\title{
EU-Russland: Hintergründe und Perspektiven einer schwierigen Beziehung
}

\author{
Heinz Timmermann*
}

Die Beziehungen EU-Russland sind in den vergangenen Jahren deutlich schwieriger geworden oder, wie manche es wahrnehmen, sogar in eine Krise geraten. Nahezu vergessen ist die Charta von Paris für ein neues Europa vom November 1990, in der sich die KSZE-Mitglieder für „Ein neues Zeitalter der Demokratie, des Friedens und der Freiheit“ engagieren wollten. Beigetragen haben zu den Problemen objektive Umstände, aber auch unterschiedliche Perzeptionen der Akteure gegenüber dem Partner. Verwiesen sei hier auf eine Reihe jüngster Kontroversen, darunter die Debatte um die US-Raketenabwehr, die Zukunft des Kosovo, das polnische Veto gegen die Aufnahme von Verhandlungen über die Neufassung des vertraglichen Verhältnisses EU-Russland, die Unterbrechung der Drushba-PipelineAbzweigung nach Litauen durch Moskau, der Streit um die Verlagerung des sowjetischen Kriegerdenkmals in Tallinn sowie die Auseinandersetzungen mit London um den Mord an Litwinenko und die Schließung von zwei britischen Kulturinstituten in Russland. Die Schwierigkeiten sind insofern verständlich, als sich beide Seiten in einem fließenden Zustand befinden und sich im Rahmen eines ,Größeren Europa' neu positionieren. All dies kompliziert die Konstituierung einer nachhaltigen Partnerschaft, die zwar weniger ist als Integration, doch mehr sein will als bloße interessengeleitete Kooperation. ${ }^{1}$

* Dr. Heinz Timmermann, Freier Mitarbeiter der Stiftung Wissenschaft und Politik, Berlin/Köln.

1 Aus der jüngsten umfangreichen Literatur zu den EU-Russland-Beziehungen seien hier erwähnt: Nadejda Arbatova: Russie-UE après 2007: le débat russe, Russie.Nei.Visions No. 20, Paris 2007, abrufbar unter: http:// www.ifri.org (letzter Zugriff: 04.03.2008); Katinka Barysch: Russia, realism and EU unity, CER policy brief, London 2007, abrufbar unter: http://www.cer.org.uk/pdf/policybrief_russia_FINAL_20july07.pdf (letzter Zugriff: 04.03.2008); Katinka Barysch: The EU and Russia: From principle to Pragmatismus?, CER policy brief, London 2006, abrufbar unter: http://www.cer.org.uk/pdf/EU_russia_barysch_final_10nov06.pdf (letzter Zugriff: 04.03.2008); Sabine Fischer: Russia and the EU - New developments in a difficult partnership, in: Friedrich-Ebert-Stiftung (Hrsg.): Partnership with Russia in Europe. Scenarios for a Future Partnership and Cooperation Agreement, Berlin 2006, S. 23-33; Sabine Fischer: Die EU und Russland, SWP-Studie 34, Berlin 2006; Thomas Gomart: Paris et le dialogue UE-Russie: nouvel élan avec Nicolas Sarkozy?, Russie.Nei.Visions No. 23, Paris 2007, abrufbar unter: http://www.ifri.org (letzter Zugriff: 04.03.2008); Thomas Gomart: France's Russia Policy: Balancing Interests and Values, in: The Washington Quarterly, Spring 2007, S. 147-155; Barbara Lippert: EU - ENP and Russia - clash or cooperation in triangle?, in: Friedrich-Ebert-Stiftung (Hrsg.): Partnership with Russia in Europe. Economic and Regional Topics for a Strategic Partnership, Berlin 2007, S. 12-35; Margareta Mommsen: Die Europäisch-Russischen Beziehungen - eine Europäische Perspektive, in: Ellen Bos/Jürgen Dieringer (Hrsg.): Die Genese einer Union der 27. Die Europäische Union nach der Osterweiterung, Wiesbaden 2008, S. 283-297; Arkadij Moshes: Priorität gesucht. Die EU, Russland und ihre Nachbarn, in: Osteuropa (Berlin) 2-3/2007, S. 21-33; Cornelius Ochmann/Andrei Zagorski: Den Stau auflösen: Die EU und Russland 2008, in: spotlight europe (Berlin) 1/2008; Alexander Rahr: Der „Kalte Krieg“ ist Geschichte, in: Internationale Politik (Berlin) 3/2007, S. 12-19; Winfried Schneider-Deters/Peter W. Schulze/Heinz Timmermann: Die Europäische Union, Russland und Eurasien. Die Rückkehr der Geopolitik, Berlin 2008; Hans-Joachim Spanger: EU-Russland: Was bleibt von der strategischen Partnerschaft?, in: Internationale Politik und Gesellschaft (Bonn) 2/2007, S. 91-113; Angela Stent: Berlin's Russia Challenge, in: The National Interest, Washington 1 March 2007, S. 46-52; Heinz Timmermann: Die Beziehungen zwischen Russland und der EU, in: Gerhard Mangott/Dmitri Trenin/Martin Senn/Heinz Timmermann (Hrsg.): Russlands Rückkehr. Außenpolitik unter Vladimir Putin, Baden-Baden 2005, S. 203-265; Christian Wipperfürth: Putins Russland - ein vertrauenswürdiger Partner? Grundlagen, Hintergründe und Praxis der gegenwärtigen russischen Außenpolitik, Stuttgart 2004. 
Anfang der 1990er Jahre hatte dies noch ganz anders ausgesehen: Nach dem Zerfall der Sowjetunion standen die EU-Russland-Beziehungen auf beiden Seiten im Zeichen hochgespannter Partnerschaftsvisionen, schienen die Konzepte beider Seiten auf ein gesamteuropäisches Ordnungsmodell hinauszulaufen. In der Europäischen Union ging man von der Vorstellung aus, das neue Russland werde sich nach einer Phase erfolgreicher Systemtransformation und zügigen Strukturwandels in den Rahmen der liberal-demokratisch geprägten Staaten und ihres Wertesystems einordnen und somit werden , wie wir'. Radikale Reformen von politischem System und Wirtschaftsordnung würden feste Fundamente für enge Interdependenzen bis hin zu einer Wertegemeinschaft legen. Entsprechende Bestimmungen in dem 1997 in Kraft getretenen Partnerschafts- und Kooperationsabkommen (PKA) schienen solche Perspektiven ebenso zu rechtfertigen wie die mit der Mitgliedschaft in OSZE und Europarat verbundene Übernahme gesamteuropäischer Werte und demokratischer Prinzipien durch Russland. ${ }^{2}$

Moskau seinerseits hegte die Erwartung, dass die Europäische Union dem neuen Russland konstruktiv begegnen, das Land materiell massiv unterstützen, es in eine gesamteuropäische Sicherheits- und Friedensordnung einbeziehen und mit ihm ein ,Gemeinsames Europäisches Haus ' teilen werde. Geprägt werde die Partnerschaft durch eine Gemeinschaft von Werten, in der frühere ideologische Kontroversen keine Rolle mehr spielen. In diesem Kontext werde die Europäische Union Russland als gleichberechtigte Großmacht und vollwertigen Partner der internationalen Staatengemeinschaft behandeln und schließlich sogar als Vollmitglied in die Europäische Union aufnehmen - so damals Jelzin und andere hochrangige Moskauer Politiker. Auf beiden Seiten waren die komplementären Perzeptionen und wechselseitigen Erwartungen auch deshalb so groß, weil die Beziehungen Russlands zur Europäischen Union und deren Mitgliedstaaten - anders als etwa im Rahmen des transatlantischen Bündnisses zu den USA - mehrdimensional angelegt wurden (Politik, Wirtschaft, innere Sicherheit, Internationales, Kultur), alle Ebenen umfassten (lokal, regional, national, international) und auf Dauer ausgerichtet waren.

Heute hat die Verdichtung der Beziehungen zur Europäischen Union aus russischer Sicht ihre Logik darin, dass Europa diejenige Region ist, mit der Russland historisch, politisch, wirtschaftlich und kulturell unter allen Weltgegenden am engsten verflochten ist. Seine Westgrenze zur Europäischen Union, die sich nach deren Erweiterung auf über 2000 km erstreckt, ist die stabilste unter all seinen Grenzen. Drei Viertel der Bevölkerung Russlands leben im europäischen Teil des Landes, sie blicken somit vorrangig in westliche Richtung; auch die Spitzenpolitiker Russlands betonen immer wieder den europäischen Charakter ihres Landes und seine historische und kulturelle Verbundenheit mit Europa, so zuletzt der Erste Stellvertretende Ministerpräsident Iwanow in einem Diskussionsbeitrag auf der Münchener Sicherheitskonferenz vom Februar 2008. Nicht zufällig genießt daher der visafreie Reiseverkehr zwischen Russland und Europa in der Bevölkerung höchste Priorität. Schließlich gibt es aus russischer Sicht eine Reihe aktueller Gründe, die für ein Nahverhältnis zur Europäischen Union und für eine Vertiefung der mehrdimensionalen Beziehungen sprechen. Dazu zählen: die Europäische Union als wichtigste Partnerin in den Bereichen Handel, Energieexporte und Modernisierungsinvestitionen; die Einbeziehung Russlands in den Ausbau transeuropäischer Netze von Pipelines, Fernstraßen, Eisenbahnen und Telekommunikationssystemen; die Vertiefung der sicherheits- und militärpolitischen Kooperation; das

2 Beschluß des Rates und der Kommission vom 30. Oktober 1997 über den Abschluß des Abkommens über Partnerschaft und Zusammenarbeit zwischen den Europäischen Gemeinschaften und ihren Mitgliedstaaten einerseits und der Russischen Föderation andererseits, in: Amtsblatt der EG, Nr. L 327 vom 28. November 1997. 
wachsende Gewicht des Euro und dessen Konsequenzen für die russisch-europäischen Wirtschafts- und Finanzaktivitäten; das zunehmende Gewicht der Union bei einer einvernehmlichen Gestaltung des ,Größeren Europa', insbesondere im Zeichen der Erweiterung der Europäischen Union nach Osten und der Intensivierung ihrer Beziehungen zu den ,Neuen Nachbarn` Ukraine, Moldau, Belarus und südkaukasischen Staaten.

Umgekehrt liegt das aktuelle Interesse der Europäischen Union an engen Beziehungen zu Russland auf der Hand. Für sie ist die europäische Großmacht Russland als Mitspieler für funktionale Zusammenarbeit unentbehrlich. Genannt werden sein weiträumiges Territorium, seine geografische Nähe, sein Nutzen- und Schadenpotenzial, seine Energieressourcen, seine relativ offenen Märkte und nicht zuletzt seine auch weiterhin vorhandenen militärischen Fähigkeiten. Als demokratisch orientierter, kooperativer und sich europäisch definierender Partner kann Russland aus europäischer Sicht erheblich zu Stabilität und Wohlstand auf dem Kontinent beitragen. Als peripherisierte und in ihrer Zuordnung unsichere Großmacht besteht dagegen die Gefahr, dass Russland Instabilität über Osteuropa hinaus nach Westeuropa exportiert. Hinzu kommt: Ohne Russland gibt es kein VN-Mandat für Friedenssicherung und Krisenmanagement, ohne Russland lassen sich viele Konflikte auf dem Alten Kontinent und in seinem Umfeld nur schwer regeln. Dies gilt für so zentrale Probleme wie Terrorismusbekämpfung, Fortsetzung von Abrüstung und Rüstungskontrolle insbesondere mit Blick auf den Vertrag über Konventionelle Streitkräfte in Europa, Verhinderung der Verbreitung von Massenvernichtungswaffen, Eindämmung von Organisierter Kriminalität sowie Umwelt- und Klimaschutz. Beide Seiten - die Europäische Union und Russland - stimmen bei allen Differenzen in ihren wechselseitigen Interessen und Wahrnehmungen in einem überein: Man braucht einander und muss entsprechend handeln. Denn, so Kanzlerin Merkel auf der Münchener Sicherheitskonferenz von 2007: „Gemeinsam mit Russland können wir viel bewegen und können wir viel erreichen. “3

\section{Interaktionsprobleme}

Trotz der Vielzahl gemeinsamer Interessen und fruchtbarer Interdependenzen ist die Partnerschaft EU-Russland in der Wahrnehmung der Beteiligten in eine Krise geraten. Zwar konnte auf einer Reihe von Feldern Konsens erzielt werden - von der Intensivierung des Energiedialogs über die Zustimmung der Europäischen Union zum WTO-Beitritt Russlands, die Ratifizierung des Kyoto-Protokolls durch Moskau, die im Ganzen positive Regelung des Kaliningrad-Transits sowie die Einbeziehung der neuen EU-Mitglieder in das PKA. Zugleich haben sich die ursprünglich mit dem PKA verbundenen Erwartungen jedoch nur teilweise erfüllt und sind vielfach in Enttäuschung und Vertrauensschwund zwischen den Partnern umgeschlagen. Beigetragen haben dazu insbesondere folgende, hier nur stichwortartig zu nennende Entwicklungen:

Beide Partner, Russland und die Europäische Union, durchlaufen einen schwierigen Prozess von Identitätssuche und Selbstfindung. Russland als Kern der UdSSR erlebte den Zerfall seines Imperiums und seinen Abstieg als Großmacht; die Ausdehnung von Europäischer Union und NATO nach Osten hat in Moskau ein grundsätzliches Misstrauen gegenüber dem Westen genährt. Inzwischen allerdings haben sich unter Putin im internationalen Beziehungssystem fundamentale, keineswegs abgeschlossene Machtverschiebungen ergeben, in deren Folge Russland die Schwächeperiode seiner reaktiven Politik der 1990er Jahre hinter

3 Rede der Bundeskanzlerin Angela Merkel auf der 43. Münchener Konferenz für Sicherheitspolitik am 10.02.2007, abrufbar unter: http://www.securityconference.de (letzter Zugriff: 04.03.2008). 
sich gelassen hat und zu einer eigenständigen, handlungsfähigen und selbstbewussten Großmacht aufgestiegen ist. ${ }^{4}$ Russland ist - anders als in der Jelzin-Periode - nicht nur frei von Auslandsschulden und darin begründeten Abhängigkeiten, sondern entwickelt sich selbst in raschem Tempo zu einem Akteur auf den internationalen Investitions- und Finanzmärkten. Seinen Einfluss in Europa macht Russland insbesondere über seine Energiemacht und über seine verbliebenen Positionen in dem von ihm nach wie vor als „,domaine réservé“ perzipierten GUS-Raum geltend. Dennoch bleibt auch weiterhin die Frage offen, wie sich Russland definieren soll: „Ein Staat aller Russen, die Schaffung einer russländischen Nation, eine postkommunistische Hegemonialmacht oder ein europäischer Staat, der Integration in die vorhandenen supranationalen Institutionen sucht." 5 Anders als in Moskau häufig wahrgenommen, ist die Europäische Union in ihrer Mehrheit (im Gegensatz zu den USA und einigen Neumitgliedern Ostmitteleuropas) jedoch keineswegs an einem schwachen Russland interessiert. Wenn in Moskau geglaubt werde, der Westen wolle Russland schwächen, so der deutsche Außenminister Steinmeier, so sei das falsch und deute auf einen Rückfall in Denkmuster des Kalten Krieges: „Europa braucht ein handlungsfähiges Russland, damit wir die gemeinsamen Herausforderungen in einer globalen Welt bewältigen können. “6 Ein Russland allerdings, das für Europa berechenbar ist und pragmatischen Interessenausgleich sucht.

Die Zusammenarbeit EU-Russland wird komplizierter und eine wechselseitige realistische Wahrnehmung schwieriger durch die Unterschiede in der Natur der Partner. Zwar versteht sich der Staatenverbund Europäische Union mit seinen jetzt 493 Millionen Einwohnern als globaler Akteur mit eigenen Interessen, was bei einer Realisierung von 25 Prozent des weltweiten Sozialprodukts sowie rund 20 Prozent des Welthandels und 45 Prozent der globalen Direktinvestitionen wirtschaftlich sicher zutrifft. Gerade mit Blick auf die Gemeinsame Außen- und Sicherheitspolitik liegt die letzte Entscheidung jedoch auch weiterhin bei den 27 Mitgliedstaaten. Nicht zu Unrecht genießt die Europäische Union daher in Russland wegen ihrer sichtbar eingeschränkten internationalen Handlungsfähigkeit nur begrenztes Ansehen. Auf der anderen Seite sehen wir einen zunehmend zentralisierten russländischen Staat, der seine Wiederkehr als Großmacht zwar - anders als in den 1930er Jahren - in Einklang mit den globalen Trends nicht revolutionär-konfrontativ betreibt, der aber auch weiterhin an überkommenen Konzeptionen wie ,Einflusssphären ' und ,Nullsummenspiel' festhält. Sein Ziel ist dabei nicht länger wie in den Jahren zuvor die Verteidigung des Status quo, sondern die aktive Mitwirkung bei der Gestaltung der internationalen Politik.

Diese Konstellation bietet Moskau die Chance, seine Interessen insbesondere im wirtschaftlichen Bereich unter Umgehung Brüssels über bilaterale Kanäle durchzusetzen (vor allem im Blick auf Deutschland, Frankreich, Italien). In der Europäischen Union wird dies häufig als Versuch zu massiver Einflussnahme auf die gemeinsame Linie der EU-27 und gelegentlich sogar als Manöver zur Spaltung der Union wahrgenommen und entsprechend kritisiert. Zu Unrecht, wie mir scheint: Zum einen hat Moskau wiederholt sein Interesse an ei-

4 Grundsätzlich dazu Außenminister Sergej Lawrow: Vnešnjaja politika Rossii: novyj etap, in: Expert (Moskau) 47, 17.12.2007.

5 So Gerhard Simon: Rußland auf der Suche nach seiner politischen Identität. Visionen und Wirklichkeiten, Berichte des Bundesinstituts für ostwissenschaftliche und internationale Studien (Köln) 33/1997, S. 8 .

6 Rede von Bundesaußenminister Frank-Walter Steinmeier auf der 42. Münchener Konferenz für Sicherheitspolitik: „Russland, Europa und die Welt - Perspektiven der Zusammenarbeit in globalen Sicherheitsfragen“, 05.02.2006, abrufbar unter: http://www.auswaertiges-amt.de (letzter Zugriff: 04.03.2008). Ähnlich seine Rede: „Die Welt in Unordnung - veränderte Machtverhältnisse, fehlende Strategien“ auf der Münchener Sicherheitskonferenz vom 09.02.2008, abrufbar unter: http://www.securityconference.de (letzter Zugriff: 04.03.2008). 
ner starken und einheitlich auftretenden Europäischen Union bekundet. ${ }^{7}$ Vor allem aber kann es niemand Moskau verdenken, wenn es entsprechende Chancen nutzt, solange Mitgliedstaaten im Zweifel nationale Interessen über gemeinschaftliche Ziele stellen und die Europäische Union selbst nicht die notwendige innere Kohärenz und Integration schafft.

Schließlich verleihen die Neumitglieder Polen und die baltischen Staaten aufgrund ihrer spezifischen historischen Erfahrungen und kollektiven Erinnerungen den Beziehungen EURussland besondere Akzente, wodurch die Russland-Perzeptionen innerhalb der Europäischen Union widersprüchlicher und komplizierter werden. So stehen den langen positiven Friedens- und Wohlstandserfahrungen im Westen , die Traumata und Erfahrungen derer gegenüber, für die der Kalte Krieg mit Fremdherrschaft und Diktatur einherging". 8 Während sich Putin anlässlich seiner Staatsbesuche in Budapest und Prag Anfang 2006 zur moralischen Schuld für die sowjetischen Militärinterventionen in Ungarn 1956 und in der Tschechoslowakei 1968 bekannte, weigert sich Moskau bis heute, durch analoge Gesten zur Entspannung mit Polen und den baltischen Staaten beizutragen. In Russland bildet der Sieg der Sowjetunion im Großen Vaterländischen Krieg auch weiterhin ein zentrales integratives Element der nationalen Identität, das ungebrochen in die postsowjetische Phase der russischen Eigenstaatlichkeit übernommen wurde.

Die Polen und Balten dagegen nehmen den sowjetischen Sieg nicht nur als Befreiung von Nazideutschland wahr, sondern auch als neue Unterwerfung (Polen) beziehungsweise als gewaltsame Einverleibung (Balten). Die Angst vor einem Wiederaufleben des russischen ,Imperialismus' ist lebendiger und politisch aktueller Bestandteil der polnischen und baltischen Erinnerungskultur. Konkret äußert sich dies etwa in der Suche nach einem Nahverhältnis zu den USA als Rückversicherung gegen perzipierte Gefahren aus dem Osten. Ein anderes Beispiel sind die unterschiedlichen Denkansätze zur Energieaußenpolitik der Europäischen Union: Während Polen perzipierten russischen Energieoffensiven mit der Bildung einer „Energie-NATO“ zu begegnen empfiehlt, favorisiert Deutschland als Instrument zu einvernehmlicher Absprache mit dem Partner in Moskau eine „Energie-OSZE“. Ganz offensichtlich wollen Polen und die baltischen Staaten ,ihre Mitgliedschaft in der EU dazu nutzen, um dem mächtigen Sukzessionsstaat Russland heute selbstbewusst entgegenzutreten “9 und Moskauer Einfluss insbesondere in ,Zwischeneuropa' zurückzudrängen.

In Russland wird die Haltung der Neumitglieder aus Ostmitteleuropa als ,primitive Russophobie“ und als „,komplexbeladener Atavismus“ (Sergej Jastrshembskij) einer Politik gedeutet, die das europäische Rad zum Schaden Moskaus zurückdrehen will und sich von Intoleranz und Konfrontation mit Moskau leiten lässt. ${ }^{10}$ Die von Polen angestrebte rasche EU-Mitgliedschaft für die Ukraine, Moldau und ein demokratisches Belarus, das mit einem Fleischembargo begründete Veto gegen Verhandlungen über einen neuen Vertrag EU-Russland, das anvisierte Sonderabkommen Warschaus mit den USA über die Errichtung eines Raketenabwehrschirms in Polen - all dies wird in Moskau (und überwiegend auch in Brüssel) als Versuch Warschaus gewertet, spezifische nationale Interessen wahrzunehmen, den eigenen Einfluss in Brüssel zu steigern, die Union wie Rumsfeld im Kontext des Irakkriegs

7 Ein Beispiel hierfür bietet Vladimir Čizov: Moskva nuždaetcja v sil’nom Evrosojuze, in: Nezavisimaja gazeta (Moskau), 22.10.2007. Der Autor ist Ständiger Vertreter Russlands bei der EU in Brüssel. Ähnlich Sergej Iwanow in einem Diskussionsbeitrag auf der Münchener Sicherheitskonferenz vom 10.02.2008, abrufbar unter: http://www.securityconference.de (letzter Zugriff: 04.03.2008).

8 So Günter Verheugen: Europa in der Krise. Für eine Neugründung der europäischen Idee, Köln 2005, S. 33-34. Der Autor ist Stellv. Kommissionspräsident.

9 Hierzu Mommsen: Die Europäisch-Russischen Beziehungen, 2008, S. 283.

10 Natal’ja Melikova: Eti ljudi privnesli v Evrosojuz duch primitivnoj russofobii, Interview mit Sergej Jastržembskij, dem Vertreter des Präsidenten für die Beziehungen Russland-EU, in: Nezavisimaja gazeta, 17.11.2004. 
in ein „Altes Europa“ und ein „Neues Europa“ zu teilen und sie damit zum Schaden ihrer Beziehungen zu Russland de facto zu spalten. ${ }^{11}$ Mit der konfrontativen Konzeption der nationalistisch orientierten Kaczynski-Brüder hat sich Polen in der Europäischen Union allerdings eher selbst isoliert. Nach Übernahme der Regierung durch die bürgerlich-liberale Koalition Tusk ist sie in wesentlichen Punkten gescheitert, wie der von Moskau ebenso wie von Warschau bekundete Wille zum Abbau der wechselseitigen Spannungen demonstriert.

\section{Gemeinsame Interessen ohne gemeinsame Werte?}

Vor dem Hintergrund zunehmender Differenzen in den jeweiligen Interessen und Perzeptionen wächst die Unklarheit darüber, was den konkreten Inhalt der ,strategischen Partnerschaft" EU-Russland ausmacht: Wird sie erst angestrebt oder ist sie bereits erreicht und weiter auszubauen? Nur soviel lässt sich vorerst sagen: In ihrer multidimensionalen Vernetzung der Partner ist sie im gemeinsamen Verständnis mehr als bloße Kooperation, wie es sie auch während des Kalten Kriegs gab, jedoch weniger als institutionelle Einbindung Russlands in die Europäische Union, die nach den Erfahrungen des Zweiten Weltkriegs zum Kern der europäischen Wertegemeinschaft und zur Friedensmacht wurde. Aus Sicht der Europäischen Union gewinnt die Partnerschaft dann feste Fundamente, wenn sie sich auf gemeinsame europäische Werte stützt und dadurch Nachhaltigkeit und integrative Impulse erhält. ${ }^{12}$

Die Moskauer Politik dagegen ist weder ideologie- noch wertegeleitet, sondern orientiert sich an den von seiner Führung definierten kulturellen Traditionen und nationalen Interessen Russlands. Integration bedeutet für Moskau aktives und gleichberechtigtes Mitwirken in globalen Organisationen wie VN, G-8 und WTO, nicht aber Übertragung nationaler Souveränität zugunsten eines supranationalen Gebildes. So gesehen gilt die Europäische Union Russland vor allem als Quelle für wirtschaftliche Modernisierung und technologische Innovation in einem Gesamteuropäischen Wirtschaftsraum („Modernisierungspartnerschaft“), nicht aber als Modell und Impulsgeber für politisch-gesellschaftliche Demokratisierung. Die Partnerschaft EU-Russland ist somit eher von vielfacher Interaktion geprägt, nicht aber von Integration in europäischem Verständnis. In diesem Kontext ist die Moskauer Führung im Sinne einer Teil-Europäisierung zwar zur Anpassung seiner Gesetzgebung an bestimmte, eher technisch definierte wirtschafts- und handelspolitische Standards, Normen und Regelwerke bereit, wie sie ja auch der angestrebte WTO-Beitritt erfordert. Auch sind - anders als zu Sowjetzeiten - Meinungsfreiheit, Reisefreiheit und das Recht auf Privatbesitz gewährleistet. Dennoch: Obwohl in der Verfassung von 1993 erstmals in der russischen Geschichte der Vorrang der Rechte und Freiheiten des Menschen gegenüber dem Staat verkündet wird, sind die Machthaber in Moskau nur in Grenzen disponiert, über verbale Bekenntnisse hinaus solche Prinzipien und Grundwerte auch tatsächlich zu realisieren, wie sie im Rest Europas verstanden und gelebt werden: Demokratie, Rechtsstaatlichkeit, Medienfreiheit, Menschenrechte.

11 Aufgrund unterschiedlicher Reaktionen auf den Irakkrieg der USA unterteilte der damalige US-Verteidigungsminister Rumsfeld die EU-Mitgliedstaaten in US-kritische „Alteuropäer“ einerseits, zu denen er die Mehrheit der Mitgliedstaaten vor der Osterweiterung von 2004 mit Deutschland und Frankreich an der Spitze zählte, und US-freundliche „Neueuropäer“ andererseits, zu denen er die Mehrheit der ostmitteleuropäischen Beitrittsländer mit Polen und den baltischen Staaten als Kern rechnete. In ihrer scharfen Gegenüberstellung hat diese Typisierung freilich nur begrenzte Aussagekraft, da sich ihr mehrere EU-Länder - z.B. Großbritannien einerseits und Ungarn andererseits - nicht eindeutig zuordnen lassen.

12 Vgl. hierzu Diana Schmidt: Eine Wertelücke zwischen Russland und dem Westen?, in: russlandanalysen (Bremen) 70/2005, S. 2-5, abrufbar unter: http://www.russlandanalysen.de (letzter Zugriff: 04.03.2008). 
Tatsächlich betrachtet die Putin-Führung die Forderung nach Übernahme europäischer Grundwerte und Demokratieprinzipien als unzulässige Einmischung in die inneren Angelegenheiten Russlands, da sie die unterschiedlichen historischen und kulturellen Voraussetzungen nicht berücksichtige. Russland versteht sich zwar als Teil Gesamteuropas. Zugleich sieht die Putin-Führung ihr Land jedoch nicht als Teil eines solchen Europa, wie es die Europäische Union verkörpert. Vielmehr ist sie entschlossen, als starker Staat und als konsolidierte, unabhängige und gleichberechtigte Großmacht in patriotisch-nationalem Konsens an den spezifischen kulturhistorischen, ethischen und systemformenden Traditionen und Werten Russlands festzuhalten und auf dieser Grundlage die inneren Entwicklungen des Landes selbst zu gestalten.

Auch die Europäische Union und ihre Mitgliedstaaten lassen sich in ihrem Verhältnis zu Russland von konkreten Interessen leiten, am deutlichsten sichtbar im Energiedialog. Sie verbinden dies jedoch mit der Erwartung, dass die interessengeleitete Politik des russischen Partners nicht in autoritär-zentralistische Traditionen zurückfällt und damit Zuverlässigkeit, Berechenbarkeit und Flexibilität infrage stellt, sondern dass sich Russland in der Partnerschaft mit der Europäischen Union an gemeinsamen Werten und demokratischen Prinzipien orientiert. Aus Sicht der Union bestimmt der russische Bezug auf europäische Werte ganz wesentlich Grad, Charakter, Qualität und Nachhaltigkeit der Partnerschaft. Je größer das Ausmaß rechtsverbindlicher Übereinkünfte und gewachsener institutioneller Bindungen, desto dauerhafter die Interdependenzen als Basis der Partnerschaft. Im Verständnis der Europäischen Union ist dies nicht mit einem Aufdrängen eigener Modelle, mit der Etablierung eines „,normativen Imperiums“ (Wladimir Putin) gleichzusetzen, wie es unter den Moskauer Eliten häufig wahrgenommen wird. Vielmehr soll damit zum Ausdruck gebracht werden: Innovation und Modernisierung können nachhaltig nur dann gelingen und die angestrebten Interdependenzen fördern, wenn sie sich bei allen Varianten von bewährten europäischen Prinzipien leiten lassen und sich nicht auf die Übernahme einzelner technischer Standards und Normen beschränken. Berechenbarkeit, Effizienz und Transparenz in Politik, Wirtschaft, Sicherheitsagenturen und sogar in den Außenbeziehungen sind dauerhaft nur dann gewährleistet - so die Überzeugung in der Europäischen Union -, wenn die Exekutive in ein funktionierendes System von Gewaltenteilung eingebunden ist, wenn Rechtssicherheit gewährleistet ist und wenn freien und unabhängigen Medien sowie einer lebendigen Zivilgesellschaft die Möglichkeit zu kritisch-konstruktiver Artikulation gegeben wird.

Folgt man der Theorie des demokratischen Friedens etwa im Sinne Czempiels, so schlagen Rezentralisierung sowie autoritäre und nationalistische Trends in der russischen Innenpolitik auf die Außenbeziehungen des Landes und folglich auch auf die EU-Russland-Beziehungen durch, gefährden dauerhaft stabile Kooperation und bergen sogar erhöhtes Konfliktpotenzial in sich. Kritische Stimmen aus der Gesellschaft und von Seiten ausländischer Partner dürfen daher nicht als Stabilitätsrisiko denunziert, sondern müssen im Gegenteil als unverzichtbare Stabilitätsfaktoren anerkannt werden. Als Ausdruck gesellschaftlicher Vielfalt und gelebter Partnerschaft bilden sie unerlässliche Instrumente der Korrektur möglicher Fehlentwicklungen.

Auch in Zukunft werden durch vielfältige Asymmetrien verursachte Ungewissheiten den Charakter der EU-Russland-Beziehungen prägen. Eine zentrale, in Europa durchaus kontrovers diskutierte Frage lautet: Wird Russland bei der Ausgestaltung seines inneren Systems im Zeichen eines staatsmachtfixierten bürokratischen Autoritarismus erneut seinen traditionellen und auf Dauer angelegten Sonderweg einschlagen, der die Partnerschaft mit der Europäischen Union auf Interessenausgleich begrenzt? Dieser Weg war bereits in der Vergangenheit unter dem letzten Zaren und unter dem Spätkommunisten Breshnew gescheitert. 
Oder handelt es sich bei dem System Putin auch in dessen Nachfolge um einen temporären, die Turbulenzen der Jelzin-Periode überwindenden Umweg, der nach Überwindung des „Rechtsnihilismus“ (Dmitrij Medwedew) ${ }^{13}$ über die Zwischenetappe einer von Putin und seinem Nachfolger souverän gesteuerten Demokratie letztlich zu einer auch wertemäßig geprägten gegenseitigen „Verflechtung und Vernetzung von Wirtschaft und Gesellschaft" ${ }^{\text {"14 }}$ führt? Aufschluss über die Perspektiven der Partnerschaft könnten die Ergebnisse der praktischen Zusammenarbeit geben, die die Partner in den Wegekarten zu den vier anvisierten „Gemeinsamen Gesamteuropäischen Räumen“ entwickelten (Wirtschaft, Inneres, Äußeres, Wissenschaft und Kultur). ${ }^{15}$ Die vier Kooperationsräume, die zur Richtschnur für verstärkte Interdependenzen wurden, sollen hier kurz in ihrer Substanz sowie im Blick auf ihre Wahrnehmung in Russland und der Europäischen Union umrissen werden.

\section{Vier Gemeinsame Gesamteuropäische Räume}

Die zu den vier Räumen führenden Wegekarten (road maps) des EU-Russland-Gipfels vom Mai 2005 listen auf 52 Seiten mehr als 400 Einzelmaßnahmen auf. Zwar ist das Dokument völkerrechtlich nicht verbindlich und nennt keine inhaltlichen und zeitlichen Prioritäten für die Realisierung der einzelnen Projekte. Dennoch wird es von beiden Seiten als nützlich und perspektivreich wahrgenommen, da es trotz Auffassungsunterschieden unter den Partnern auf praktischen Feldern bei allen divergierenden Wertevorstellungen einen umfangreichen Katalog konkret abzuarbeitender Aufgaben nennt.

Der Gemeinsame Wirtschaftsraum EU-Russland mit seinen offenen, integrierten Märkten gilt beiden Seiten als Kern und Triebkraft der strategischen Partnerschaft und in seiner Komplementarität als zentraler Impulsgeber für gegenseitige Verflechtung und Interdependenz. So gehen bei einem Gesamtvolumen von 231 Milliarden US-Dollar im Jahre 200657 Prozent der Exporte Russlands in die Europäische Union und 6 Prozent der EU-Exporte nach Russland. Rund 70 Prozent aller Auslandsinvestitionen in Russland stammen aus der Europäischen Union; umgekehrt machen die Investitionen Russlands in Europa nur den zehnten Teil aus, zeigen aber rasch steigende Tendenz. Angestrebt wird, durch wechselseitige Abmachungen die Handels- und Wirtschaftsaktivitäten zu erleichtern, den Marktzugang und das Investitionsklima zu verbessern, eine hohe Regulierungskompetenz zu erreichen, einen zuverlässigen Rechtsrahmen zur Sicherung von Stabilität und Berechenbarkeit zu schaffen und die Infrastrukturen zu entwickeln. Auf diesen Feldern besteht zwischen den Partnern eine Disposition zu weitgehender Harmonisierung, zumal der bevorstehende und von der Europäischen Union unterstützte Beitritt Russlands zur WTO Wirtschaftsreformen stimuliert, Transparenz fördert und größere Rechtssicherheit schafft. Sollten die Interdependenzen in einem allmählichen Prozess sämtliche Wirtschaftsbereiche erfassen, so könnte Russland nach dem Romano-Prodi-Motto „Alles außer Institutionen“ perspektivisch in ein Verhältnis

13 Rede vor dem Bürgerforum in Moskau vom 22.1.2008, abrufbar unter: http://kreml.org/opinions/171436199 (letzter Zugriff: 07.03.2008), sowie Rede vor dem Wirtschaftsforum in Krasnojarsk vom 15.2.2008, abrufbar unter: www.newsru.com/russia/15feb2008/medved4i7z_print.html (letzter Zugriff: 07.03.2008).

14 Frank-Walter Steinmeier: Verflechtung und Integration. Eine neue Phase der Ostpolitik der EU: Nicht Abgrenzung, sondern Vernetzung lautet das Gebot der Globalisierung, in: Internationale Politik 3/2007, S. 6-11, hier S. 8 .

15 Wegekarten zur Schaffung von vier Gemeinsamen Räumen: Wirtschaft; Freiheit, Sicherheit und Justiz; äußere Sicherheit; Forschung, Bildung und kulturelle Aspekte, abrufbar unter: http://ec.europa.eu/external_relations/ russia/summit_05_05/index.htm (letzter Zugriff: 10.03.2008). Ausführlich hierzu Hannes Adomeit/Rainer Lindner: Die „Gemeinsamen Räume“ Rußlands und der EU. Wunschbild oder Wirklichkeit, SWP-Studie 34, Berlin 2005. 
zur Europäischen Union treten, das demjenigen Norwegens im Rahmen des Europäischen Wirtschaftsraums (EU plus EFTA) ähnelt.

So ist Russland aus der Sicht der Europäischen Union mittlerweile ,von einem unzuverlässigen Kostgänger zum Markt der unbegrenzten Möglichkeiten“ geworden, nachdem es „das permanente Krisenmanagement in einen soliden Wachstumspfad münden ließ“. ${ }^{16}$ Mit den neuen Möglichkeiten sind zugleich jedoch auch neue Probleme in den EU-Russland-Beziehungen entstanden, insbesondere im Blick auf wechselseitige Investitionen in strategischen Wirtschaftsbereichen der Partner. In Deutschland und einigen weiteren EU-Ländern wächst der Widerstand gegen entsprechende Beteiligungen Russlands, wobei der Telekommunikationssektor und die europäische Luft- und Raumfahrtindustrie (Stichwort EADS) im Mittelpunkt stehen. Ähnliches gilt für den Energiebereich, in dem zwecks Verhinderung monopolistischer Marktmachtbildung ausländischer Konzerne Produktion, Durchleitung und Verteilung voneinander getrennt werden sollen. Hintergrund sind Befürchtungen, Moskau könne seine Beteiligungen als Druckmittel für politische Zwecke nutzen. Immerhin hat Gasprom bis heute in wenigstens 16 der 27 EU-Mitgliedstaaten investiert und in den drei größten Gasmärkten einen gewissen Zugang zu den Konsumenten erhalten.

In Russland wird die Einschränkung für Auslandsinvestitionen als ungerechtfertigter Eingriff in die Investitionsfreiheit im Allgemeinen und als Benachteiligung russischer Investoren im Besonderen wahrgenommen. In der Tat: Auslandsinvestitionen sind keine Einbahnstraße. Allerdings hat auch Moskau Beschränkungen von Investitionen in strategischen Industriebereichen und beim Zugang zu den Bodenschätzen vorgenommen - nicht zuletzt in Abwehr perzipierter EU-Bestrebungen, einseitig westliche und so auch Brüsseler Ordnungsvorstellungen durchzusetzen und die asymmetrischen Beziehungen der Jelzin-Periode zu perpetuieren. Aus europäischer Sicht müssen solche die Interdependenz konterkarierende Maßnahmen Ausnahmeregelungen bleiben, die klar definiert sind und bereits bestehende Investitionen nicht antasten. Zwar konnten sich die Partner auf ihrem Gipfel von Samara vom Mai 2007 darauf einigen, gemeinsam konkrete Regelungen zur Investitionssicherheit zu treffen, doch bleibt das umstrittene Problem der Bedingungen wechselseitiger Investitionen auch weiterhin offen.

In diesem Kontext haben die ,Energiekriege“ in der Ukraine Anfang 2006 und ein Jahr darauf in Belarus im EU-Raum hohe Wellen geschlagen sowie in der Wahrnehmung der Europäer Image und Reputation Russlands als verlässlichen Energielieferanten beschädigt. Immerhin liefert Russland 30 Prozent seiner Erdölimporte und 50 Prozent seiner Erdgasimporte in die Europäische Union. Gewiss verfolgt Russland mit seinem Energiepotenzial auch politische Ziele. Das lässt sich am Beispiel Belarus gut demonstrieren. So fügt sich die hartnäckig betriebene und schließlich erreichte Umwandlung des Minsker Pipelinesystems Beltransgas in ein paritätisches russisch-belarussisches Joint Venture in das Moskauer Bestreben, die Pipelinesysteme im postsowjetischen Raum und darüber hinaus zu übernehmen und sich damit die Herrschaft über die Energieströme aus dem GUS-Raum in Richtung Europa zu sichern. ${ }^{17}$

Unzutreffend ist jedoch der im EU-Raum vernehmbare Vorwurf an Moskau, die Energiepreise für Belarus (und andere GUS-Partner) aus vornehmlich politischen Erwägungen heraus dramatisch erhöht, in der Transitfrage wenig Verlässlichkeit gezeigt und bewusst einen

16 So Hans-Joachim Spanger: Paradoxe Kontinuitäten. Die deutsche Russlandpolitik und die koalitionären Farbenlehren, HSFK-Report (Frankfurt) 12/2005, S. 19.

17 Vgl. hierzu und zum Folgenden meinen Beitrag: Der Energiekrieg Russland-Belarus: Ursachen und Folgen, in: Reinhard C. Meier-Walser (Hrsg.): Energieversorgung als sicherheitspolitische Herausforderung, München 2007, S. 153-162. 
Vertrauensbruch gegenüber seinen europäischen Vertragspartnern begangen zu haben. Denn zum einen hat die Europäische Union in den Verhandlungen über den russischen WTO-Beitritt Moskau immer wieder dazu gedrängt, von politischen Preisen schrittweise zu Weltmarktpreisen überzugehen - auch in den Beziehungen zu ihren GUS-Partnern. Zum anderen war es der belarussische Präsident Lukaschenko, der Russland durch wiederholte illegale Energieentnahme zu vorübergehender Schließung der durch Belarus führenden Leitungssysteme veranlasste und damit zugleich die europäischen Energiekonsumenten traf. Im Westen wenig wahrgenommen, trug der ,Energiekrieg ' paradoxerweise zu Entwicklungen bei, die dort eigentlich grundsätzlich begrüßt werden: „Der Übergang zu Weltpreisen auf den Energiemärkten bedeutete die Absage an die frühere imperiale Politik wirtschaftlicher Subventionen im Tausch gegen politische und politisch-militärische Loyalität." 18

Auch in Zukunft wird der Energiekomplex immer wieder Probleme zwischen der Europäischen Union und Russland aufwerfen. Denn zum einen konnte sich die Union bislang trotz wiederholter Ansätze nicht auf eine einheitliche Energiepolitik einigen. Und zum anderen verweigert sich Russland (und ähnlich übrigens auch Norwegen) bislang einer Öffnung seiner Pipelinesysteme für ausländische Benutzer. Dies blockiert nicht nur die Verabschiedung der Energiecharta, sondern hindert die Europäer darüber hinaus daran, durch Diversifizierung der Energielieferungen etwa aus Zentralasien einseitige Abhängigkeiten zu vermeiden. Die von den Partnern Russland und Europäische Union angestrebte (und bis Ende 2007 durch das polnische Veto durchkreuzte) Neufassung des PKA soll daher nach EU-Vorstellungen den Rahmen für engere Kooperation im Energiebereich fixieren, wie sie ansatzweise bereits in der Wegekarte „Wirtschaft" dargelegt wurde. Wir brauchen einen Energiedialog, so Außenminister Steinmeier Anfang 2007, , der die Interessen von Produzenten, Konsumenten und Transitländern ausgleicht und einen Streitschlichtungsmechanismus einschließt" ${ }^{19}$ Ein Durchbruch bei der Realisierung dieser Ziele würde der anvisierten Verflechtung EU-Russland kräftige Impulse geben. Davon sind wir jedoch vorerst noch weit entfernt. Die in Samara erzielte Übereinkunft, wonach die Europäische Union künftig frühzeitig über mögliche Lieferstörungen informiert werden soll, ist ein zwar wichtiger, aber nur ein kleiner Schritt in diese Richtung.

Im Hinblick auf den Gemeinsamen Raum Freiheit, Sicherheit und Justiz hatte Moskau auf dem Haager Gipfel vom November 2004 an einem verengten Verständnis von Freiheit als ,Reisefreiheit“ festgehalten und damit die Verabschiedung des gesamten Dokuments der vier Wegekarten blockiert. Aus Moskauer Sicht gehörte der Aspekt „Grundwerte und demokratische Prinzipien" nicht in diese Rubrik, sondern bildete einen Teil des wesentlich unverbindlicheren politischen Dialogs EU-Russland. In Europa wurde diese Interpretation als unzutreffend angesehen, bildete doch der Hinweis auf die Bedeutung der Grundwerte für die Partnerschaft einen integralen Bestandteil des PKA. In einer erstaunlichen Wende akzeptierte Russland schließlich auf dem Moskauer Gipfel vom Mai 2005 in verschiedenen Zusammenhängen der Wegekarten - allerdings nicht wie im PKA in der Präambel - die erweiterte EU-Interpretation von „Freiheit“, einschließlich seiner zentralen Elemente Demokratie, Rechtssicherheit, Menschen- und Minderheitenrechte sowie Medienfreiheit. Wie bereits eingangs betont, wurde im Folgenden freilich deutlich: Nicht zuletzt im Zeichen des Konstrukts der „Souveränen Demokratie“ klaffen das Verständnis der Grundwerte und der Charakter ihrer Realisierung eher weiter auseinander und setzen die Partnerschaft immer wieder neuen

18 Aleksej Arbatov: Is A New Cold War Imminent?, in: Russia in Global Affairs (Moskau) 3/2007, S. 84-97, hier S. 91.

19 Steinmeier: Verflechtung und Integration, 2007, S. 10. 
Belastungen aus. Putins Hinweis, in schwierigen Situationen an den Institutionen vorbei zum ,handgesteuerten System“ überzugehen, kann die Skepsis der Europäer nur vertiefen. ${ }^{20}$

Die zumindest verbale Konzession Moskaus in der Interpretation von „Freiheit“" erfolgte, so scheint es, vornehmlich aus zwei Gründen. Zum einen verstehen die Russen in ihrer Mehrheit unter „Freiheit“" in erster Linie soziale Sicherheit und Gleichheit vor dem Gesetz, weniger aber die von der Putin-Führung eher kritisch gesehene Freiheit, sich autonom zu organisieren und unabhängig von den Regierenden für selbstdefinierte politische Ziele einzutreten. Der Verweis der Regierenden auf die aktuell herrschende „Stabilität“ hat diese spezifische Konnotation und ist daher zum zentralen Slogan zur Sicherung ihrer Macht geworden. Das in Europa oft übersehene spezifische Verständnis von Freiheit reduziert die Bereitschaft der Menschen, sich für einen demokratischen Aufbruch zu engagieren und entsprechende ,Farbenrevolutionen“ für politischen Wandel einzuleiten. Es birgt also für die Staatsmacht wenig Explosivstoff, zumal die Opposition nur schwach organisiert und von persönlichen Ambitionen zerrissen ist. Und zum anderen erfolgte die Konzession von Moskau deshalb, um Fortschritte in der Frage des langfristig anvisierten visafreien Reiseverkehrs zu erzielen. Dies ist ein Punkt, dem von der Bevölkerung Russlands große Bedeutung beigemessen wird und dessen Realisierung daher die Zustimmung der Bevölkerung zum System Putin zusätzlich festigt. Tatsächlich einigten sich die Partner auf ihren Gipfeln von London (2005) und Sotschi (2006) auf einen Kompromiss, der einzelnen Berufsgruppen aus Wissenschaft, Kunst und Bildung die visafreie Einreise in die Europäische Union erlaubt. Vor allem aber kombiniert er russische Wünsche nach Reiseerleichterungen und europäische Wünsche nach Abschluss eines Abkommens zur Rücknahme solcher Migranten, die von Russland aus illegal nach Europa gelangten. Sollten die Abmachungen realisiert werden und schließlich sogar den visafreien Reiseverkehr Russland-EU möglich machen, so wäre dies ein zentrales Element wechselseitiger positiver Wahrnehmung der Partner und damit ein wichtiger Baustein zur Errichtung eines ,Gemeinsamen Europäischen Hauses‘.

Die Wegekarte Äußere Sicherheit ist die unverbindlichste aller road maps und weist den höchsten Grad von Unbestimmtheit auf. Im Blick auf die einzelnen Projekte werden weder Prioritäten und Mechanismen zu ihrer Umsetzung genannt noch werden zeitliche Rahmen vorgegeben. Als zentrale Ziele gelten gemeinsames Krisenmanagement und Schaffung eines größeren Europa ohne Trennlinien, basierend auf gemeinsamen Werten. Ähnliches gilt für das Bekenntnis zu „wirksamem Multilateralismus“ und zur „Stärkung der Rolle und der Wirksamkeit entsprechender internationaler und regionaler Organisationen“.

Tatsächlich beziehen Europäische Union und Russland, wie bereits eingangs angedeutet, in den internationalen Beziehungen auf wichtigen Feldern gleiche oder ähnliche Positionen. Zentrale Stichworte sind: effektiver Multilateralismus in einem funktionierenden System internationaler Regeln und Institutionen, in dem die VN den Kern bilden, sowie gewaltfreie Konfliktlösungen im Nahen und Mittleren Osten (Israel/Palästina beziehungsweise Iran), einer hochexplosiven Region im Umfeld von Europäischer Union und Russland. „Wir müssen uns weiter um engere Beziehungen zu Russland bemühen, das einen wichtigen Faktor für unsere Sicherheit und unseren Wohlstand bildet", heißt es dazu in der Europäischen Sicherheitsstrategie vom Dezember 2003. ${ }^{21}$ Noch deutlicher präzisierte das Strategiepapier des Berliner Auswärtigen Amts vom August 2006: „Eine gesamteuropäische Friedensordnung

20 Zitiert nach Heinrich Vogel: Machtwechsel als Hütchenspiel, in: russlandanalysen (Bremen) 154/2007, S. 2-5, hier S. 3, abrufbar unter: http://www.russlandanalysen.de (letzter Zugriff: 07.03.2008).

21 Europäischer Rat: Ein sicheres Europa für eine bessere Welt. Europäische Sicherheitsstrategie, Brüssel 2003, S. 14. 
und die Lösung wichtiger sicherheitspolitischer Probleme von Baltikum bis nach Nahost läßt sich nur mit und nicht ohne Russland erreichen." 22 In diesem Kontext wollte Sergej Iwanow in seinen Diskussionsbeitrag auf der Münchener Sicherheitskonferenz vom Februar 2008 die Bildung gemeinsamer Friedensmissionen nicht ausschließen, sofern ihr Inhalt sowie ihr zeitlicher Rahmen klar definiert seien.

Die erwähnte Unbestimmtheit und Unverbindlichkeit der Wegekarte „Äußere Sicherheit“ verweist jedoch zugleich darauf: Unter dem Dach gemeinsamer Absichtserklärungen und Ziele existiert eine Reihe unterschiedlicher Positionen und Wahrnehmungen, die die EURussland-Beziehungen in den vergangenen Jahren auch auf internationalem Felde komplizierten und den angestrebten effektiven Multilateralismus auszuhöhlen drohen. So tragen die US-Pläne zur Errichtung von Komponenten eines bilateral vereinbarten Raketenabwehrsystems in Polen und Tschechien an der NATO vorbei ebenso wie die Suspendierung des Vertrags über Konventionelle Streitkräfte in Europa durch Moskau dazu bei, die Beziehungen zwischen den Partnern zu belasten und Konflikte in und über Europa auszutragen. Indem USA und NATO ihre Stützpunkte und Infrastruktur immer näher an Russlands Westgrenze heranführen, bleibt wenig von der Zusicherung der NATO, nach der deutschen Wiedervereinigung und der NATO-Osterweiterung Russland an seinen Westgrenzen einen sicheren Flankenschutz gegenüber weiteren geopolitischen Veränderungen und militärischen Vorwärtsstrategien zu bieten. Hinzu kamen weitere Divergenzen, die das Vertrauensklima zwischen Europäischer Union und Russland abkühlen ließen:

Es gelang den Partnern nicht, sich im Rahmen der VN auf den zukünftigen Status des Kosovo zu einigen: Während die meisten EU-Mitglieder in Anlehnung an den AhtisaariPlan für eine international überwachte Unabhängigkeit des Separatistengebildes eintraten und diese im Februar 2008 auch offiziell anerkannten, verweist Russland auf die VN-Resolution 1244, die das Kosovo als integralen Bestandteil Serbiens definiert. Die durchaus nachvollziehbare Position Moskaus dürfte nicht allein auf die Unterstützung für den traditionellen Verbündeten Belgrad und den Versuch zur Einflusswahrung auf dem Balkan zurückzuführen sein, wie es in Europa überwiegend wahrgenommen wird. Ganz wichtig für das Verständnis der russischen Position ist die Perspektive, dass nach dem von der VN nicht sanktionierten NATO-Angriff auf Serbien 1999 nunmehr eine weitere Entscheidung unter Umgehung der VN gefällt werden soll - nicht legal, doch legitim, wie es in Europa gesehen wird. In Moskau wird dies als Schwächung der VN und damit der eigenen Positionen und Interessen wahrgenommen, zumal dort die Ereignisse von 1999 in Politik und Gesellschaft noch heute als Trauma politischer Isolierung und Marginalisierung empfunden werden.

Ein zweites Beispiel für divergierende Interessen EU-Russland sind die ,eingefrorenen Konflikte' in den Separatistengebilden Transnistrien, Südossetien und Abchasien, und dies trotz der Tatsache, dass beide Seiten sie als ernsthafte Bedrohung für die eigene Sicherheit wahrnehmen. Seit ihrer Osterweiterung 2004 und der damit einhergehenden Einbeziehung von Moldau und Georgien in die Politik der ,Neuen Nachbarschaft` dringt die Europäische Union auf eine Lösung der Konflikte und erklärt ihre Bereitschaft, sich beim Krisenmanagement stärker als bisher zu engagieren. Russland zeigt daran jedoch wenig Interesse, und dies nicht zuletzt deshalb, um angesichts der näherrückenden Europäischen Union und des Engagements von USA und NATO in Georgien mit dem Konzept fortwirkender, eingefrorener Konflikte“ und andauernder ,kontrollierter Instabilität‘ seinen Einfluss in der Region zu

22 Zum Inhalt des offiziell nicht publizierten Papiers siehe: Frankfurter Allgemeine Zeitung: Berlin schlägt in der EU-Russlandpolitik eine „Annäherung durch Verflechtung“ vor, 04.09.2006. 
wahren. Solche Absichten sowie die vagen Warnungen aus Politik und Medien Russlands, im Falle der internationalen Aufwertung des Kosovo seinerseits die genannten Separatistengebilde als Völkerrechtssubjekte anzuerkennen - all dies ist geeignet, die Reputation Moskaus in der Region und darüber hinaus die Partnerschaft mit der Europäischen Union zu beschädigen.

In der Wahrnehmung der russischen Eliten wächst die Vorstellung, ihr Land sei von einer ihm feindlich gesinnten Welt umgeben. Der trotz massiver Moskauer Einflussnahme erfolgte demokratische Aufbruch in der Ukraine 2004 hat diese Perzeption weiter zugespitzt. Russische Verschwörungstheorien und Einkreisungsängste veranlassten seine Führung zu einer zunehmend kritischen Haltung gegenüber der OSZE und bewegten sie sogar dazu, 2005 vorübergehend die Zahlung der Beiträge zum OSZE-Haushalt einzustellen. Als die russischen Behörden bei den Staatsdumawahlen 2007 und den Präsidentschaftswahlen 2008 die Aktivitäten der OSZE-Wahlbeobachtermission scharf eingrenzten (und damit auch die Kritik der Europäischen Union hervorriefen), verzichtete die OSZE und deren Büro für demokratische Institutionen und Menschenrechte (ODIHR) sogar ganz auf Teilnahme. In der Wahrnehmung Moskaus verwendet die Organisation zunehmend doppelte Standards zu Lasten der Länder im GUS-Raum, um die auf Europa orientierte Opposition bei Wahlen durch politische Manipulation und massive materielle Unterstützung zu beeinflussen und sie damit für einen Macht- und Seitenwechsel weg von Russland zu präparieren. Von daher brauche die OSZE dringend eine umfassende Reform, heißt es in Moskau, bei der der Schwerpunkt von der zivilgesellschaftlich-demokratischen Förderung hin zur sicherheitspolitischen und wirtschaftlichen Dimension verschoben werde. Die Europäische Union wehrt sich zwar nicht gegen eine stärkere Betonung dieser beiden Aspekte, hält jedoch grundsätzlich an der Bedeutung der Wahlbeobachtung für die Realisierung europäischer Demokratieprinzipien im postsowjetischen Raum fest. Wenn man bedenkt, dass Europäische Union und OSZE im Hinblick auf Weiterentwicklung und praktische Umsetzung europäischer Prinzipien eng zusammenwirken, so bildet das schwindende Interesse und Engagement Moskaus im Rahmen der OSZE kein gutes Signal für die Partnerschaft EU-Russland.

\section{Integrationskonflikte in ,Zwischeneuropa“?}

Gerade das letzte Beispiel demonstriert: Unterschiedliche Wahrnehmungen der Absichten des Partners können die Verständigung zwischen ihnen konterkarieren und das Konfliktpotenzial steigern. Am deutlichsten zeigt sich dies im Blick auf die Länder ,Zwischeneuropas“ Ukraine, Moldau und Belarus, die in Moskau als „Nahes Ausland“ und in Brüssel als „Neue Nachbarn“ bezeichnet, jedoch nicht als gemeinsamer Nachbarschaftsraum wahrgenommen werden. ${ }^{23}$ Den Ländern ,Zwischeneuropas ' wurde zunehmend bewusst, dass es für sie mit der Europäischen Union in der Region einen machtbalancierenden Faktor und sogar eine Alternative zu Russland als ihrem bislang zentralen Bezugspunkt gibt. In diesem Bewusstsein wurden sie dadurch bestärkt, dass Moskau seinen GUS-Partnern kein attraktives Gesellschaftsmodell und kein positives Integrationsprojekt auf der Basis angemessener Mit-

23 Unter der breiten Literatur zur Nachbarschaftspolitik seien hervorgehoben: Anne Faber: Europäische Nachbarschaftspolitik und externe Demokratieförderung durch die EU im Spiegel der Forschung, in: integration 1/2008, S. 89-94; Andrei Zagorski: Common European Neighbourhood and the Post Soviet Space, in: Friedrich-Ebert-Stiftung (Hrsg.): Partnership with Russia in Europe, Berlin 2007, S. 36-51; Lippert: EU - ENP and Russia, 2007; Barbara Lippert: Teilhabe statt Mitgliedschaft? Die EU und ihre Nachbarn im Osten, in: Osteuropa 2-3/2007, S. 69-94; Dmitri Trenin: Russia, the EU and the common neighbourhood, London 2005, abrufbar unter: http://www.cer.org.uk/pdf/essay_russia_trenin_sept05.pdf (letzter Zugriff: 04.03.2008). 
entscheidung bietet. Ambitionen in Richtung auf demokratisch-rechtsstaatliche Verhältnisse in den Ländern ,Zwischeneuropas ' werden in Russland als Kontrollverlust und potenzielle Niederlage im Nullsummenspiel gegen die Europäische Union gewertet. Wo liegen im Kern die unterschiedlichen Interessen und Wahrnehmungen?

Zunächst: Die Europäische Union zielt mit ihrer Nachbarschaftspolitik weder auf geopolitisches Ausgreifen noch auf Absorption ihrer osteuropäischen Nachbarn nach dem Muster eines Nullsummenspiels. Tatsächlich geht es ihr darum, an ihrer Ostflanke politisch stabile, wirtschaftlich prosperierende, rechtsstaatlich verfasste und damit berechenbare Partner zu haben. Dabei drängt die Europäische Union weder wie die USA auf einen von außen gesteuerten und geostrategisch motivierten Regime Change noch strebt sie wie Russland nach Sicherung eines Status quo, der loyale autoritäre Regime stützt. Zwar wird die Europäische Union einen Anspruch Russlands, in ,Zwischeneuropa“ als bestimmende Ordnungsmacht zu fungieren, nicht akzeptieren. Angesichts der engen wirtschaftlichen sowie der vielfachen historischen, menschlichen und kulturellen Verbindungen dieser Länder zu Russland ist die Europäische Union jedoch zugleich bestrebt, Interessen und Perzeptionen Russlands in ihr Kalkül einzubeziehen und entsprechenden Einfluss auf ihre „Neuen Nachbarn“ zu nehmen. Bezeichnenderweise hält sich die Unterstützung Brüssels für die US-finanzierte und gegen russischen Einfluss gerichtete regionale GUAM-Organisation in Grenzen.

Russland dagegen perzipiert die Länder ,Zwischeneuropas “ als vorrangig russische Einflusszone und hält bis heute an seiner „Mittelfristigen Strategie gegenüber der EU“ von 1999 fest. ${ }^{24}$ Danach soll die Entwicklung der Partnerschaft mit der Europäischen Union dazu beitragen, „Russlands Rolle als führende Kraft bei der Bildung eines neuen Systems politischer und wirtschaftlicher Beziehungen im GUS-Raum zu konsolidieren“. Russland versteht seinen Einflussverlust in der Region und den parallel dazu wachsenden Einflussgewinn der Europäischen Union als geopolitische Expansion der Union mit dem Ziel, über die Implantation farbiger Revolutionsviren die Machtverhältnisse im postsowjetischen Raum umzustürzen und Russland aus seinem „Nahen Ausland“ zu verdrängen. Nur vereinzelt sind in Moskau auch solche Stimmen zu vernehmen, die die Annäherung der Länder ,Zwischeneuropas ‘ an die Europäische Union nicht vorrangig auf deren Expansionsambitionen zurückführen, sondern auf die Attraktivität des Gesellschaftsmodells der Union. „Unlike the Soviet Union with its ideology of world Communism or imperial Russia with security guarantees as with Pan-Slavism and Orthodoxy, the Russian Federation does not have an attractive project to offer these days. " 25

Vor diesem Hintergrund wollen sich Europäische Union und Russland laut Wegekarte „Äußere Sicherheit“ ganz allgemein über die Zukunft ,Zwischeneuropas“ verständigen, die neutral als ,,an EU und Russland grenzende Region“ bezeichnet wird. Für beide Seiten haben im fragilen Beziehungsdreieck EU-Russland-,Zwischeneuropa' wechselseitige stabile Beziehungen und einvernehmliches Krisenmanagement letztlich Vorrang vor gewaltgestützten geopolitischen Ambitionen zu Lasten der anderen Seite. Mit dem Westen sei Konkurrenz angesagt, nicht aber Konfrontation - so kürzlich Außenminister Lawrow. Umgekehrt stößt eine von den USA betriebene rasche NATO-Mitgliedschaft der Ukraine und Georgiens oder gar deren Einbeziehung in das erwähnte Raketenabwehrsystem unter vielen ,Altmitgliedern“ der Europäischen Union - darunter auch Deutschland - auf große Skepsis, und dies nicht zuletzt deshalb, weil sich Russland damit zusätzlich eingekreist und provoziert

24 Die Mittelfristige Strategie in: Diplomatièeskij vestnik (Moskau) 11/1999, S. 20-28.

25 So Arkady Moshes: Reaffirming the Benefits of Russia's European Choice, in: Russia in Global Affairs 3/2005, abrufbar unter: http://eng.globalaffairs.ru (letzter Zugriff: 04.03.2008). 
fühlen müsste. Das gilt umso mehr angesichts der US-Pläne, Militärstützpunkte in Rumänien und Bulgarien zu installieren.

Angesichts des labilen Zustands in den Ländern ,Zwischeneuropas" ist nicht auszuschlieBen, dass die Europäische Union und Russland künftig bei Gestaltung und Perspektiven dieses Raums über die Integrationskonkurrenz hinaus in Konflikt geraten - nicht zuletzt über jeweils unterschiedliche Wahrnehmungen der dortigen innenpolitischen Entwicklungen im Allgemeinen und die Übernahme europäischer Demokratienormen mit ihren Folgen für die internationale Zuordnung im Besonderen. Die Integrationskonkurrenz könnte sich in einem negativen Szenario dann zuspitzen, wenn sich Russland und einzelne Länder ,Zwischeneuropas ' in Interpretation und Anwendung europäischer Grundwerte stark auseinanderentwickeln. Umgekehrt wäre in einem positiven Szenario auf längere Sicht sogar daran zu denken, die auf Russland bezogenen vier Gemeinsamen Räume mit den in der Europäischen Nachbarschaftspolitik verankerten Aktionsplänen zu harmonisieren und damit der Schaffung eines ,Größeren Europa ' wichtige Impulse zu geben. Dies setzt freilich voraus, dass sich beide Seiten über gemeinsame Grundzüge ihrer inneren Strukturen und ihrer Politik in ,Zwischeneuropa' verständigen.

\section{Annäherung durch Verflechtung?}

In den vergangenen 15 Jahren hat sich zwischen der Europäischen Union und Russland ein enges und intensives Netzwerk von belastbaren politischen und wirtschaftlichen Interessen herausgebildet. Aus europäischer Sicht ist eine dauerhafte strategische Partnerschaft mit Russland jedoch nur dann möglich, wenn sie die Realisierung gemeinsamer europäischer Grundwerte ins Auge fasst, auf wechselseitigem Vertrauen beruht und den offenen Dialog über kontroverse Punkte nicht scheut. Mit der Neuauflage des Sonderwegs im Zeichen einer autoritären Dynamik würde sich Russland im gesamteuropäischen Raum selbst isolieren nicht nur gegenüber EU-Europa, sondern angesichts möglicher zukünftiger demokratischer Aufbrüche auch im postsowjetischen Raum Osteuropas und des Südkaukasus. Zugleich ergibt sich hieraus für die europäische Politik ein gewisses Dilemma. Denn einerseits hat sich Putins autoritär-bürokratischer, systemstabilisierender Etatismus insbesondere auf wirtschaftlichem Gebiet als eine wichtige Bedingung für die Fortentwicklung der europäischrussischen Interessenallianz erwiesen. Andererseits fordert er die demokratische Glaubwürdigkeit der Europäer heraus und gefährdet auf längere Sicht auch die Interessenallianz, da nachhaltige politische Kooperation, vertiefte wirtschaftliche Interdependenz und dauerhaft gesicherter Frieden in der Wahrnehmung der Europäer nicht von der auf europäischen Prinzipien gründenden demokratischen Ordnung zu trennen sind.

In diesem Kontext tut sich die Europäische Union angesichts unterschiedlicher Erfahrungen, Interessen und Wahrnehmungen ihrer Mitgliedsländer schwer, einen kohärenten Ansatz gegenüber Russland zu finden. Grob gesehen zeichnen sich zwei unterschiedliche Strategien ab, die sich - zugespitzt ausgedrückt - als Strategie des containment beziehungsweise als Strategie des engagement charakterisieren lassen. Die Strategie der Eindämmung wird am deutlichsten von Polen und den baltischen Staaten betrieben. Sie geht davon aus, dass eine enge Verzahnung EURussland die Übernahme demokratischer Prinzipien und eine Beendigung geopolitischer Ambitionen durch Moskau insbesondere in ,Zwischeneuropa' voraussetzt. Der Wechsel von der national-populistischen Regierung Kaczynski zu der liberal-konservativen Regierung Tusk führt Polen ganz offensichtlich wieder näher an den mainstream der EU-Russlandpolitik heran, wie nicht zuletzt die Wiederaufnahme hochrangiger bilateraler Besuche in Moskau und Warschau demonstriert. Dass Polen dabei mit Ronald Tusk statt einer ,strategischen Partnerschaft“ eher die 
Bildung einer ,harmonischen Koexistenz“ mit Russland ins Auge fasst, ${ }^{26}$ verweist zugleich auf fortbestehende Vorbehalte in Warschau und könnte dazu beitragen, die Ausarbeitung eines neuen EU-Russland-Vertrags zu erschweren. Die EU-Mitglieder Westeuropas zeigen zwar Verständnis für historisch bedingte Vorbehalte ostmitteleuropäischer Partner. Jedenfalls sind sie entschlossen, ,nicht über ihre Köpfe hinweg zu entscheiden“ (Merkel), sondern sie zu konsultieren und ihre Interessen zu berücksichtigen. Dies müsse jedoch auch in der Gegenrichtung funktionieren: „Nur wenn neue und alte Mitgliedsstaaten bei der Schaffung einer neuen EU-RusslandPolitik zusammenarbeiten, läßt sich eine Balance der Interessen innerhalb der EU finden “. ${ }^{27}$

Die „Altmitglieder“ ihrerseits praktizieren eine Strategie des engagement - freilich in vielen inhaltlichen Varianten. Das Verhältnis Großbritanniens zu Russland hat sich jüngst deutlich abgekühlt - genannt seien der eingangs erwähnte Fall Litwinenko und seine Folgen, der Druck auf die in Russland tätigen britischen Energieunternehmen BP und Shell sowie die Verschlechterung der NATO-Russland-Beziehungen, die sich negativ auch auf die Haltung Großbritanniens zu den EU-Russland-Beziehungen auswirkt. Italien hat seine positive Einstellung zu Russland nach Berlusconi grundsätzlich auch unter Prodi beibehalten und dürfte daran unter jedem Nachfolger festhalten. Ein wichtiger Grund hierfür sind die engen, rasch anwachsenden Wirtschaftsbeziehungen, symbolisiert insbesondere in der Zusammenarbeit zwischen dem Energieunternehmen ENI und Gasprom. Das Frankreich Sarkozys schließlich hat seine unter Chirac betriebenen Sonderbeziehungen zu Russland in den Hintergrund gedrängt zugunsten einer Neubelebung seiner transatlantischen Beziehungen sowie seiner regionalen Aktivitäten zur Bildung einer ,,Mittelmeerunion“ “. ${ }^{28}$ Auch hier sind es ganz wesentlich wirtschaftliche Überlegungen, die das Interesse Frankreichs an einer Einbindung Russlands dennoch stimulieren. So erhielt der französische Energiekonzern Total die Konzession, sich an der Ausbeutung der Stockman-Gasvorkommen in der Barentssee zu beteiligen, was noch kurz zuvor westlichen Firmen verweigert worden war.

In Deutschland herrscht parteiübergreifend Konsens über die Notwendigkeit, die strategische Partnerschaft mit Russland auszubauen, ohne darüber - wie Kanzler Schröder - auf Kritik an den demokratischen Defiziten und Repressionen des System Putin gegenüber zivilgesellschaftlichen Akteuren zu verzichten. ${ }^{29}$ Von Schröders Charakterisierung Putins als „lupenreinen Demokraten“ haben sich sowohl Merkel als auch Steinmeier ausdrücklich distanziert. So ist Merkels Rhetorik zwar zurückhaltender als diejenige ihres Vorgängers, und gelegentlich halten Christdemokraten ihrem sozialdemokratischen Koalitionspartner vor, in seiner Russlandstrategie die Bedeutung der Anwendung demokratischer Werte und Prinzipien nicht deutlich genug hervorzukehren. Insgesamt jedoch weist die Russland-Politik der Kanzlerin, eingebettet in häufige Bezüge auf die strategische Partnerschaft mit Russland, keine prinzipiellen Unterschiede zu derjenigen ihres Vorgängers auf. Ähnlich wie Steinmeier hält der CDU-Politiker Andreas Schockenhoff, der Koordinator für die deutsch-russische gesellschaftliche Zusammenarbeit im Auswärtigen Amt, eine enge Zusammenarbeit EU-Russland für alternativlos und sieht in Russland sogar einen ,potentiellen Wertepartner“. Seine Begründung: ,Russland ist aufgrund seiner Lage und seiner Größe, seines politischen und militärischen Gewichts, seines Energiereichtums und seines wirtschaftlichen Po-

26 Donald Tusk: Unser Nachbar Russland, in: Frankfurter Allgemeine Zeitung, 18.2.2008.

27 So die Bundestagsabgeordnete Angelica Schwall-Düren: Wir brauchen eine Neue Europäische Ostpolitik für Russland, in: Friedrich-Ebert-Stiftung (Hrsg.): Partnerschaft mit Russland in Europa, Berlin 2005, S. 16-23, hier S. 21.

28 Vgl. Gomart: Paris et le dialogue UE-Russie, 2007.

29 Hierzu im einzelnen Matthes Buhbe: Grundzüge einer deutschen Russland-Strategie, in: Friedrich-Ebert-Stiftung (Hrsg.): Kompass 2020. Deutschland in den internationalen Beziehungen. Ziele, Instrumente, Perspektiven, März 2007, Berlin; sowie meinen Beitrag: Die deutsch-russischen Beziehungen im europäischen Kontext, in: Internationale Politik und Gesellschaft 1/2007, S. 101-121. 
tentials für die EU von strategischer Bedeutung." Um dem näherzukommen, brauchten Deutschland und die Europäische Union Geduld und einen langen Atem. Von dem neuen Präsidenten Medwedew erwartet Schockenhoff die Einleitung „tiefgreifender Reformen“ und fordert dazu auf, ihm einen ,Vertrauensvorschuss“ zu gewähren. ${ }^{30}$

In diesem Kontext sehen die EU-,,Altmitglieder" mehrheitlich gerade wegen der vielen gemeinsamen Interessen und einer potenziellen Annäherung von Werten im Dialog mit Russland Chancen, Probleme zu lösen und Perspektiven zu entwickeln. Aus ihrer Sicht dürfen die vielfachen Interessen, die Russland und die Europäische Union verbinden, nicht durch überzogene Konditionalität belastet werden. Vielmehr sollte deren Erfüllung Ergebnis eines längerfristigen Prozesses im Zeichen von Pragmatismus, neuem Realismus und Respekt für die schwierigen Transformationsprozesse in Russland sein. So wird die wachsende Einbeziehung Russlands in den Weltmarkt und sein steigendes Investitionsengagement im Ausland reziproke westliche Aktivitäten in Russland erleichtern und dabei EU-Spielregeln wie Transparenz, Corporate Governance und Umweltstandards fördern, die weniger von politischer Einflussnahme als von kommerziellem Kalkül gekennzeichnet sind. Bei alldem geht die Europäische Union in ihrem Engagement von der gegenwärtigen russischen Wirklichkeit aus und nicht von den unrealistischen Hoffnungen und Erwartungen der 1990er Jahre. Die europäischen Werte als dauerhafte Grundlage für nachhaltige Partnerschaft werden zwischen den Partnern zwar auch weiterhin thematisiert, nicht zuletzt unter Berufung auf die russischen Selbstverpflichtungen gegenüber Grundprinzipien von OSZE und Europarat. Zugleich bildet ihre mangelnde Implementierung jedoch kein prinzipielles Hindernis für die Realisierung gemeinsamer Interessen, wie sie mit der Regelung des explosiven Konflikts während des demokratischen Aufbruchs in der Ukraine im Dezember 2004 beispielhaft gelang. Im Rahmen mehrerer Verhandlungsrunden unter Teilnahme des EU-Trios Solana/Kwasniewski/Adamkus sowie des Staatsduma-Präsidenten Gryslow und des OSZEGeneralsekretärs Kubis gelang damals der Durchbruch, bei dem sich Juschtschenko und Janukowytsch auf ein Sieben-Punkte-Programm einschließlich einer Wiederholung der zuvor manipulierten Wahlen einigten. Aus kooperativer Wahrnehmung gemeinsamer Interessen EU-Russland, darunter Fortschritten bei der Bildung einer Freihandelszone und bei der Gewährung eines visafreien Reiseverkehrs, sollen allmählich gemeinsame Werte erwachsen. Damit setzt die Europäische Union auf sozialisierende Wirkung kleiner Verflechtungen und Harmonisierungsschritte, wobei gemeinsame Werte nicht als Voraussetzung für die strategische Partnerschaft gelten, sondern ähnlich wie bei Korb 3 der KSZE als Referenz für den Dialog.

Konkret heißt dies: In ihrem Verhältnis zu Russland wird sich die Europäische Union wohl auch in Zukunft kritisch-konstruktiv verhalten, in ihren Zielen berechenbar bleiben und legitime Interessen Moskaus respektieren. Dies geschieht nicht zuletzt in dem Bewusstsein, dass die Instrumente der Europäischen Union für eine effektive Politik der Konditionalität und damit für ihre tatsächlichen Einflusschancen auf Entscheidungsfindung und inneren Wandel in Russland gering sind. Die nach europäischen Protesten erfolgte partielle Entschärfung der Gesetzgebung zu den Nichtregierungsorganisationen 2005 ist eher die Ausnahme. In ihrer großen Mehrheit gibt die russische Bevölkerung - in Europa nur unzureichend wahrgenommen - Stabilität, zivilen Lebensperspektiven und moderater Wohlstandssteigerung Vorrang vor demokratischer Selbstbestimmung und Mitwirkung. Radikaler Kritik der Europäischen Union an der „,manipulierten Demokratie“ und dem autoritären System Putin stehen

30 Andreas Schockenhoff: Medwedew einen Vertrauensvorschuss geben, in: Frankfurter Allgemeine Zeitung, 29.02.2008. 
die Menschen eher skeptisch gegenüber. Die Begriffe „Demokratie“ und „Marktwirtschaft“ sind nach der schwierigen Transformationsphase unter Jelzin weitgehend diskreditiert. Mit ihrer überwiegenden Unterstützung des System Jelzin und seinen Fehlentwicklungen insbesondere im Blick auf den wirtschaftlichen Kollaps 1998 hat westliche Kritik in Russland unter den Menschen einiges an Glaubwürdigkeit und Überzeugungskraft eingebüßt. ${ }^{31}$

Vor diesem Hintergrund drängte die Europäische Kommission Anfang 2004 darauf, ,,von großen politischen Erklärungen zu einer themenbezogenen Strategie und Agenda überzugehen “. ${ }^{32}$ Insofern setzt die Union auf einen unsichtbaren Wertetransfer, ${ }^{33}$ angestoßen durch politische Öffnung, soziale Differenzierung und die Herausbildung einer selbstbewussten Mittelklasse in Russland, durch internationale wirtschaftliche Verflechtung und durch eine Vielzahl von internationalen Partnerschaften und persönlichen Begegnungen jenseits der gouvernementalen Ebene. Die Erfahrungen mit dem KSZE-Prozess zeigen - freilich unter anderen Bedingungen -, dass enge partnerschaftliche Kooperation langfristig auch die politische Kultur verändern kann. Grundsätzlich setzt die Strategie der Verflechtung dabei nicht die Existenz gemeinsamer Werte voraus, sondern dient dazu, diese längerfristig zu erreichen. „Der schrittweise und schwierige Weg Russlands in Richtung Demokratie und effektive Verteidigung von Bürgerrechten wird westlichen Augen weiterhin ungewöhnlich erscheinen“ - so Marc Franco, der Leiter der EU-Delegation in Moskau. „Doch in einer breiteren historischen Perspektive wird die Mitgliedschaft Moskaus im Europarat Wirkung zeigen." ${ }^{34}$

Nachdem Polen sein Veto gegen eine vertragliche Neufassung der Beziehungen EURussland aufgehoben hat, werden in naher Zukunft entsprechende Verhandlungen beginnen, zumal die Krise in den europäisch-russischen Beziehungen die Europaorientierung der Putin-Administration nicht grundsätzlich beschädigt hat. In dem neuen, den aktuellen Bedingungen angepassten und völkerrechtlich verbindlichen Vertrag, der eher pragmatisch-operativ als normativ geprägt sein dürfte, werden die Partner unter Einschluss der bislang fehlenden Dimensionen Inneres und Internationales zwar ihre Kooperation und Interdependenz stärken, zugleich jedoch auf gleichberechtigter Grundlage als eigenständige Zentren ihre Unabhängigkeit wahren. In den Augen der Europäischen Union gilt Russland damit nicht länger wie die „Neuen Nachbarn“ ,Zwischeneuropas“ als transformationsgeprägter Juniorpartner, der in seinen Beziehungen zur Europäischen Union verschiedenen Formen der Konditionalität unterliegt und Asymmetrien zu akzeptieren hat.

Deutschland wird dabei laut Strategiepapier des Auswärtigen Amts auf ein ,umfassendes, integrativ angelegtes und nach vorn weisendes Abkommen“ hinwirken - und ,dies mit klaren Signalen, dass Russland in Europa willkommen ist“. Dabei würden in den „Vertrag über Strategische Partnerschaft" - so könnte seine Bezeichnung lauten - die aktualisierten Absichtserklärungen der vier Wegekarten in ihrer Substanz integriert und in den Details im Anhang beigefügt oder in separaten Abkommen niedergelegt, beispielsweise im Blick auf den Komplex Energie. Um dem Vertrag Dauer und Nachhaltigkeit zu verleihen, darf aus europäischer Sicht der Bezug auf gemeinsame europäische Werte und Grundprinzipien

31 Vgl. hierzu Gerhard Mangott: Werte oder Interessen. Die Beziehungen der EU mit Russland oder gesinnungsethische Illusionen, in: russlandanalysen (Bremen) 121/2006, abrufbar unter: http://www.russlandanalysen.de (letzter Zugriff: 04.03.2008).

32 Europäische Kommission: Communication from the Commission to the Council and the European Parliament on relations with Russia, COM (2004) 106, 09.02.2004.

33 Gerhard Simon: Allianz für die Freiheit oder Potemkinsche Dörfer? Russland und der Westen nach dem Ende des Kalten Krieges, in: Europäische Rundschau (Wien) 4/2002, S. 49-65, hier S. 64.

34 The Moscow Times, 26.10.2007, S. 8. 
- freilich ohne bindende Konditionalitätsklauseln - nicht fehlen. ${ }^{35}$ Nicht nur das gewachsene Selbstbewusstsein Russlands verweist darauf, dass dies nicht einfach sein wird. Auch die Tatsache, dass neben neuen EU-Mitgliedern wie Polen auch Altmitglieder wie Frankreich und Großbritannien im Rahmen der Neuakzentuierung ihrer Russlandpolitik spezifische Vorstellungen zu den EU-Russland-Beziehungen entwickelten, wird die Vertragsverhandlungen kompliziert gestalten und den Vertrag selbst voraussichtlich auf den kleinsten gemeinsamen Nenner fixieren.

\section{Perspektiven}

Es ist zu hoffen, dass Russland unter der Präsidentschaft Medwedew den wechselseitigen Interessen durch stärkere Bindung an gesamteuropäische Werte und deren schrittweise Realisierung größere Nachhaltigkeit verleihen wird. Denn eine von Rezentralisierung des Staates und Eliminierung sämtlicher Gegengewalten geprägte Stabilität schafft letztlich Instabilität, an der die Europäische Union keineswegs interessiert ist. Auch sind Verschwörungstheorien wenig geeignet, den für eine funktionierende Partnerschaft EU-Russland notwendigen und auch von Moskauer Seite angemahnten „Gemeinsamen Raum des Vertrauens“ zu schaffen. ${ }^{36}$ Meist dienen sie dazu, von eigenen Fehlern abzulenken, die eigene Schuld der Einkreisungsstrategie eines verständnislosen und feindlichen Auslands anzulasten und damit im eigenen Lande zugleich nationalistische und fremdenfeindliche Gefühle zu wecken, nicht zuletzt im Verhältnis zu den Europäern. Ein eindrucksvolles Beispiel für die pauschale Verleumdung seiner politischen Gegner bietet die scharfe Wahlkampfrede Putins vom November 2007 im Sportpalast des Lushniki-Parks, mit der er als Listenführer der Partei Einheitliches Russland vor 5000 seiner Anhänger auftrat. ${ }^{37}$

Die Zukunft der EU-Russland-Beziehungen wird zum einen ganz wesentlich von dem sich formierenden Charakter der Partner bestimmt. Wird sich die Europäische Union mit der Ratifizierung des Lissabonner Reformvertrags als eigenständiger, handlungsfähiger internationaler Akteur profilieren und gegenüber Russland einheitliche Positionen beziehen? Und wird Russland unter Präsident Medwedew den wechselseitigen Interessen durch stärkere Bindung an gesamteuropäische Werte größere Nachhaltigkeit verleihen? Eine Antwort auf diese Fragen wird erst die Zukunft geben können.

Zum anderen sind es wechselseitige Interessen und Wahrnehmungen, die die Beziehungen weiterhin determinieren werden. Aus Sicht der Europäischen Union bilden gemeinsames internationales Konfliktmanagement, Zusammenarbeit im Wirtschafts- und Energiebereich und zivilgesellschaftliche Kontakte zentrale positive Kooperationsfelder. Zugleich jedoch wird die wachsende Präsenz Russlands auf der Weltbühne gerade auf dem Energiesektor vielfach als einseitige Instrumentalisierung seiner wachsenden Macht perzipiert. Erschwerend kommt hinzu, dass eine gemeinsame Lösung der ,eingefrorenen Konflikte ebenso wenig in Sicht ist wie einvernehmliche Positionen über die Zukunft ,Zwischeneuropas‘. Für Russland bleibt die Europäische Union auf längere Sicht bei allen Problemen ein

35 Zur Diskussion über einen neuen EU-Russland-Vertrag vgl. Nadezhda Arbatova: Russia-EU-Quandry, in: Russia in Global Affairs 2/2006, S. 100-110; die Beiträge von Katinka Barysch, Fyodor Lukyanov und Sabine Fischer in: Friedrich-Ebert-Stiftung (Hrsg.): Partnership with Russia in Europe, Berlin 2006; sowie die Ergebnisse des von Sergej Karaganov angestoßenen Workshops „Russia’s European Strategy: A New Start“, in: Russia in Global Affairs 3/2005, S. 8-21.

36 So Konstantin Kossatschow: Miteinander, nebeneinander, gegeneinander?, in: Berliner Zeitung, 16.11.2004. Der Autor ist Vorsitzender des Auswärtigen Ausschusses der russischen Staatsduma.

37 Die Rede findet sich in: Rossijskaja gazeta - Nedelja (Moskau), abrufbar unter: http://www.rg.ru/2007/11/22/ putin-forum.html (letzter Zugriff: 07.03.2008). 
zwar unbequemer, doch notwendiger Präferenzpartner für das ambitiöse Modernisierungsprojekt. Weder Medwedew noch Putin möchten in die Geschichte als Präsidenten eingehen, die „Europa für Russland verloren haben“. Zugleich wendet sich Russland jedoch gegen Interpretationen, wonach Moskau seine zunehmende Stärke zu offensiver Machtentfaltung nutzt und die Europäer daher entsprechende Gegenstrategien entwickeln müssten. Dies wird als Versuch wahrgenommen, den wachsenden Einfluss Moskaus einzudämmen, das Land geopolitisch einzukreisen und den Partner nachhaltig zu schwächen. In diesem Kontext wirft die erwähnte Ausarbeitung des PKA-Folgeabkommens als Grundlage für die zukünftigen EU-Russland-Beziehungen neue Probleme auf. Immerhin verweist die Absicht der Partner zur Neufassung des Dokuments auf ihr fortbestehendes Interesse an einer vertraglichen Fundierung ihrer Beziehungen. Tatsächlich könnte der neue Vertrag dazu beitragen, Misstrauen und Perzeptionsdifferenzen abzubauen, gemeinsamen Projekten einen profilierten Rahmen zu geben und damit den besonderen Charakter ihrer Beziehungen im Blick auf die Bildung eines ,Größeren Europa‘ zu unterstreichen.

Präsident Medwedew hat in seinen Auftritten zur Präsidentschaftswahl das Thema „Europa“ sowie ,,internationale Beziehungen“ überhaupt fast gänzlich ausgeklammert. Zugleich hat er mit seinem bemerkenswert deutlichen Akzent auf demokratische Umgestaltung und wirtschaftliche Reformen Russlands eine sichtbare Orientierung in Richtung Europa vorgegeben. Im einzelnen nannte er dabei unter anderem die Gewährung umfassender bürgerlicher Freiheiten, die Überwindung des „Rechtsnihilismus“ zugunsten der Unabhängigkeit der Justiz und der Gleichheit der Bürger vor dem Gesetz, die Meinungsfreiheit und die Unabhängigkeit der Medien, den Respekt des Privateigentums, das Zurückdrängen hoher Staatsfunktionäre aus Vorständen und Aufsichtsräten staatlich kontrollierter Unternehmen. ${ }^{38}$ Bereits ein Jahr zuvor hatte Medwedew auf dem Weltwirtschaftsforum in Davos die Ansicht vertreten, demokratische Staaten seien schlichtweg effektiver als nichtdemokratische Staaten, denn ,sie stützen sich auf die Prinzipien der Marktwirtschaft, die Herrschaft des Gesetzes und die Rechenschaftspflicht der Staatsmacht gegenüber der Gesellschaft" "39 All dies waren Stichworte, an die Kommissionspräsident Barroso und Bundeskanzlerin Merkel, die als erster ausländischer Staatsgast nach den Wahlen nach Moskau eingeladen wurde, in ihren Glückwunschschreiben an den neugewählten Präsidenten Medwedew anknüpften.

Gewiss, das Programm Medwedews enthält zunächst kaum mehr als verbale Zusicherungen, wie sie in wichtigen Aspekten wiederholt auch von Putin zu hören waren. Würden sie indes auch nur ansatzweise realisiert, so würde dies dem Kurs auf wechselseitige Verflechtung EU-Russland neue Anstöße geben, wie er in den vier Wegekarten vorgezeichnet ist. Zunächst allerdings muss offenbleiben, ob es dem in der russischen Geschichte bislang einmaligen Konstrukt eines Tandems in Form der Doppelherrschaft Medwedew/Putin überhaupt gelingt, die fragile Balance zwischen den verschiedenen Gruppen der russischen Elite zu wahren und in welche Richtung sie sich schließlich neigt - zu den Kräften von Sicherheit und Militär (Silowiki) oder zu den Vertretern liberaler Prägung. Erst danach wird sich zeigen, ob wir einer neuen, aus der russischen Geschichte wohlbekannten „Zeit der Wirren“ entgegengehen oder ob die von Medwedew vorgegebene Linie umfassender liberaler Reformen Chancen auf Realisierung hat und der strategischen Partnerschaft EU-Russland frische Impulse zu geben vermag.

38 Die wesentlichen Teile der Rede Medwedews vor dem Wirtschaftsforum von Krasnojarsk am 15.2.2008 finden sich unter: www.newsru.com/russia/15feb2008/medved4i7z_print.html (letzter Zugriff: 04.03.2008).

39 Medwedew beschwört in Davos Russlands Demokratie, in: Russland-Aktuell 29.1.2007, abrufbar unter: http:// www.aktuell.ru/russland/Politik/artikel_3272print.html (letzter Zugriff: 07.03.2008). 